\title{
Kinematic analysis of a novel 2-d.o.f. orientation device
}

\author{
J.R. Serracín $\quad$ L.J. Puglisi $\quad$ R. Saltaren $\quad$ G. Ejarque J.M. Sabater-Navarro $\quad$ R. Aracil
}

\begin{abstract}
A B S T R A C T
This paper presents the development of a new parallel robot designed for helping with bone milling surgeries. The robot is a small modular wrist with 2 active degrees of freedom, and it is proposed to be used as an orientation device located at the end of a robotic arm designed for bone milling processes. A generic $2 U \underline{P S}-1 S$ kinematic geometry is proposed for this device. This first article shows the developments on the workspace optimization and the analysis of the force field required to complete a reconstruction of the inferior jawbone. The singularities of the mechanism are analyzed, and the actuator selection is justified with the torque requirements and the study of the force space. The results obtained by the simulations allow building a first prototype using linear motors. Bone milling experiment video is shown as additional material.
\end{abstract}

\section{Introduction}

For decades, robotic technology has aroused interest among surgeons, and specially in last ten years, we are continuously finding new devices for surgical applications. Some of them are miniaturized robots for cooperative working [1], or robots with physicochemical perception $[2,3]$ or with spatial navigation abilities [4]. Society is demanding a technological evolution of surgical instruments and procedures [5]. The classic tools of open surgery are being transformed into new devices whose mission is the evolution toward a new improved surgeon, i.e. a surgeon that is able to carry out his work faster, more efficiently and with fewer errors up to this moment. In this time we have changed from open surgery to minimally invasive surgery (MIS), and from MIS to the robotic minimal invasive surgery (MIRS). Currently, this evolution is trying to improve the present robotic systems with better kinematic capacities for better movement inside the human body obtaining faster and more efficient procedures.

Surgical robots are being used widely in several areas, in some cases to improve the surgeon's accuracy to carry out sensitive tasks in reduced spaces [5]. The robots that have been used in surgical assistance include, among others, Zeus [6], da Vinci [7], or the UCB/UCSF [8] that are minimally invasive laparoscopic robots. Other surgical robotic systems as Robodoc, which is a robotic system for orthopedic procedures, or the Neuromate system [9], which is an assistant robotic system for neurosurgery. An excellent review with more examples can be found at Kazanzides et al. [10].

From a point of view of the kinematic structure of the mechanism, there are three kinds of robots; some of them are serial, some are parallel, and the others are hybrids [11]. Serial robots have been the most widely used in the medical field, however parallel robots have certain features that allow this kind of robots to take an advantage over serial ones, i.e. ability to manipulate loads greater than its own weight, high rigidity and very low weight or high speeds of operation [12]. Currently you can find applications of parallel surgical robots like vitreous retinal [13], neurosurgery [14], minimally invasive neurosurgery [15], bone drilling [16] or laparoscopic surgery [17].

Taking into account the above, our working group has addressed the development of parallel robots for applications in surgery and medical imaging 3D reconstruction. The group is currently working on the development of a 5 DOF robotic arm with a haptic based interface for the $3 \mathrm{D}$ reconstruction. This work is specially designed for skull-maxillofacial surgical applications.

This work presents a robotic mechanism based on a parallel structure, which will be used as wrist in a surgical robotic arm. It will be able to perform typical tasks of a surgical procedure such as milling, drilling, assistance in mounting screws for fixation of prosthesis in bones, etc. One of the main features of this device is its reduced dimension, which is very valuable since this feature allows you to locate the device ergonomically, freeing work space for the assistant surgeon or nurseries.

The paper is structured as follows. Section 2 presents the design of the mechanism, the kinematic and workspace analysis and the 

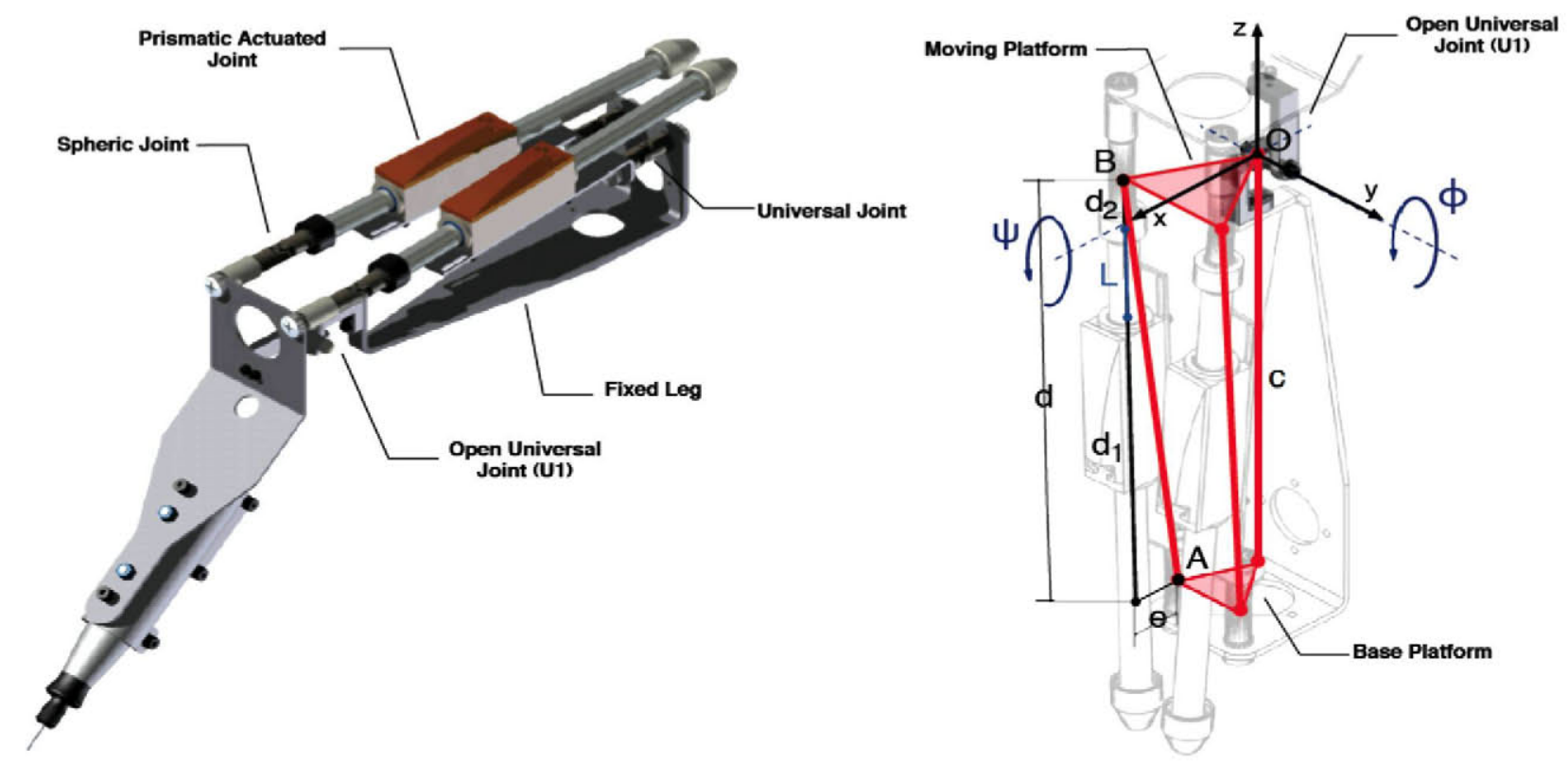

Fig. 1. Left: $C A D$ model of the mechanism. Right: schematic diagram of the mechanism in its rest configuration, showing the degrees of freedom of the device.

equations needed for singularity analysis are detailed. Section 3 presents the results and simulation plots made with equations of Section 2, and finally, Section 4 presents the first build prototype and conclusions of the work.

\section{Materials and methods}

\subsection{Design of mechanism $2 U \underline{P} S-1 U$}

$2 U P S-1 U$ mechanism consists of two platforms, one is fixed and another mobile. Both are linked by three legs (Fig. 1). Two of the legs are composed of by a prismatic joint, which is linked to the base platform through a universal joint and to the mobile platform through a spherical joint, forming a UPS (Universal-PrismaticSpherical) kinematic chain, meaning the underlying $\underline{P}$ that the actuated joint is the prismatic one. The third leg is a fixed leg, that links the two platforms via an open universal joint, to which we refer as main universal joint or $U 1$.

Applying the Grübler criterion [11], it can be shown that there are two degrees of freedom for this mechanism.

$F=\lambda(n-1)-\sum_{i=1}^{j} c_{i}$

where:

- $\lambda=6$ degrees of freedom of the whole workspace

- $n=6$ number of links of the mechanism (fixed + mobile $+2 * 2$ prismatic)

- $j=7$ number of binary joints

- $c_{i}$ constraints imposed by joint " $i$ " ( $c_{\text {universal }}=4, c_{\text {prismatic }}=5$, $c_{\text {spherical }}=3$ )

- $F=2$ degrees of freedom of the mechanism.

So the special configuration of the 2UPS - $1 U$ platform makes this device an orientation mechanism with two degrees of freedom.

\subsection{Inverse kinematics of the 2 UPS-1U device}

Being the fixed reference system $O_{x y z}$ and the mobile reference system $P_{u v w}$ (Fig. 1-right) that is solidary with the movement of the mobile platform. Both reference systems are located at the intersection of the axes of $U 1$. You can write the following vectorial equation:

$\overline{B_{i} A_{i}}=\overline{O B_{i}}-\overline{O A_{i}}$

whereas the orientation of the mobile platform referred to the fixed system $O_{x y z}$ is expressed in terms of the rotation ${ }^{\circ} \operatorname{Rot}_{p}$ matrix and defining $\overline{B_{i} A_{i}}=c_{i}$, expression 2 can be rewritten as:

$c_{i}={ }^{o} \operatorname{Rot}_{p} \times{ }^{p} B_{i}-{ }^{0} A_{i}$

where:

- ${ }^{p} B_{i}$ : corresponds to the point of insertion of the " $i$-th" leg on the mobile platform, referred to the system $P_{u v w}$.

- ${ }^{0} A_{i}$ : corresponds to the point of insertion of the " $i$-th" leg fixed platform (base), referred to the system $O_{x y z}$.

Noting the scheme presented in Fig. 1, it follows that the command for the prismatic actuators $\left(L_{i}\right)$ is:

$L_{i}=\sqrt{\left\|c_{i}\right\|^{2}-e^{2}}-\left(d_{1}+d_{2}\right)$.

\subsection{Workspace analysis}

The workspace of an orientation mechanism is defined by all possible orientations of the equivalent spherical joint. The term "possible" indicates that the restrictions imposed by the actuators, passive joints and/or geometric and physical characteristics of the mechanism are satisfied.

Given the characteristics kinematic mechanism (two degrees of freedom) Euler angles is used to represent possible orientation. This allows presenting the results on 2D graphs for easy interpretation.

Next, the constraints imposed by the joints are modeled, and the methodology is described.

\subsubsection{Model of the physical limits of prismatic actuators}

The prismatic actuators have a limited stroke; therefore the state of each actuator must be found within the physical limits of the actuator. That is to say that each actuator must satisfy:

$L_{\min } \leqslant L_{i} \leqslant L_{\max }$. 

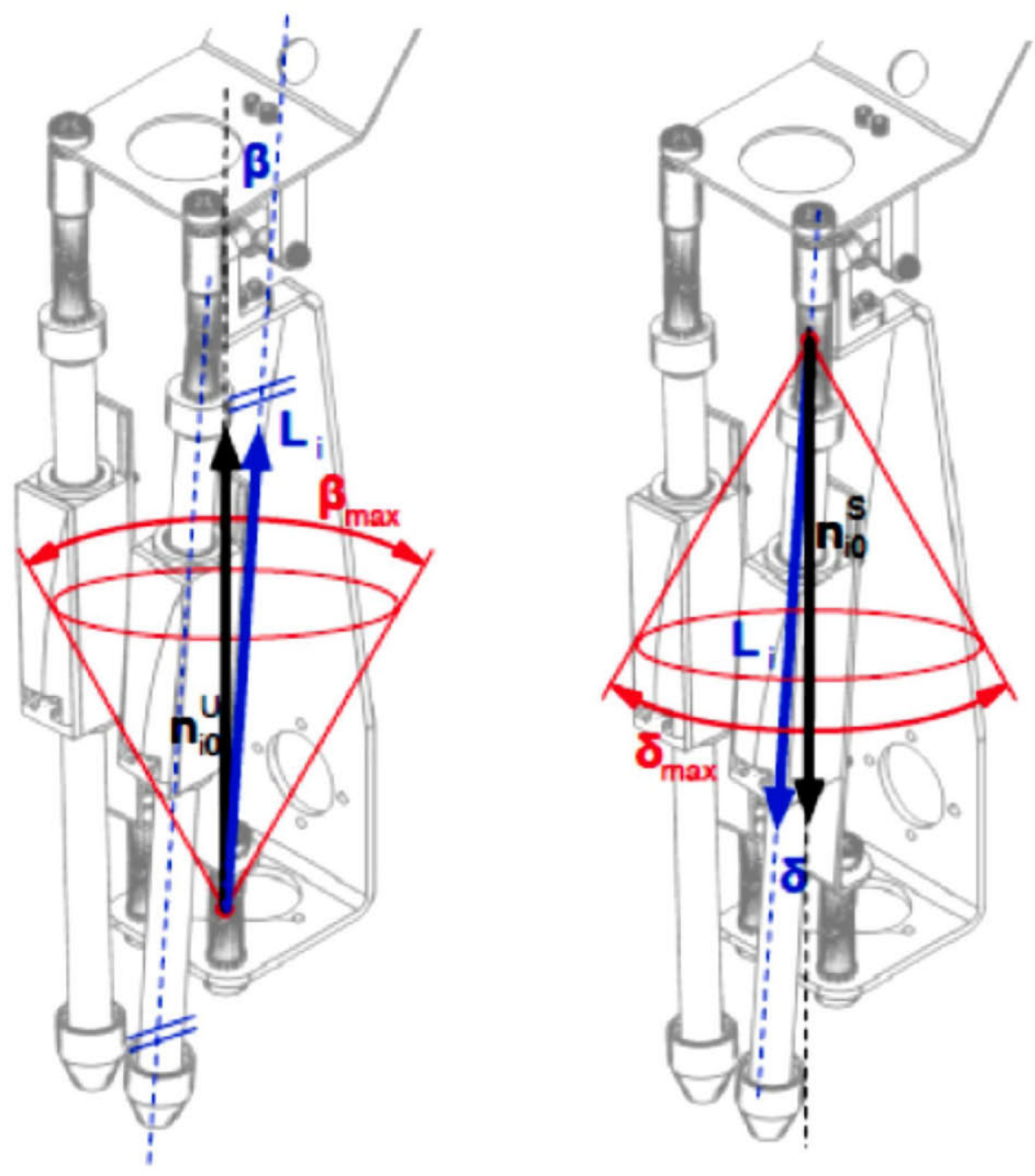

Fig. 2. Schematic diagram of the spherical and universal constraints (left and right, respectively).

\subsubsection{Model of the physical limits of universal joints}

The most usual way to model the restriction of a universal joint is to consider that the motion of the normal vector to the axes of the joint (named $n_{i}^{U}$ ) is located within a cone of opening angle $\beta_{\max }$ and generatrix $n_{i 0}^{U} . n_{i 0}^{U}$ is the vector normal to the axis of the joint when it is at rest (Fig. 2).

Particularly in this case, the normal vector to the axes of the articulation $n_{i}^{U}$ corresponds to vector direction of the fixed leg $L_{i}$ and $n_{i 0}^{U}$ corresponds to the normal vector to the fixed platform.

Therefore, the limit of the $U$ joint must verify that the angle formed between $L_{i} \mathrm{y} n_{i 0}^{U}$ is smaller than $\frac{\beta_{\max }}{2}$, i.e.:

$|\beta| \leqslant \frac{\beta_{\max }}{2}$

where

$\beta=\arccos \left(\frac{\hat{L_{i}} \cdot n_{i 0}^{U}}{\| \hat{L_{i}\|\cdot\| n_{i 0}^{U} \|}}\right)$

where the symbol $\left({ }^{\wedge}\right)$ denotes that it is a unit vector, which is obtained from dividing the vector by its norm:

$\hat{L}_{i}(\varphi, \psi)=\frac{L_{i}}{\left\|L_{i}\right\|}$.

\subsubsection{Model of the physical limits of spherical joints}

A spherical joint can be thought of as a universal joint that allows an additional rotation about an axis normal to the universal joint shafts. Therefore, the limits of the movement of the spherical joint are modeled in the same way that the universal joint. In other words, its movement is located within a cone defined by an angle of opening $\delta_{\max }$, and a generatrix that has the same direction as the normal vector to the first two lines of the spherical joint at rest (called $n_{j 0}^{S}$ ), which corresponds to the normal vector to the mobile platform.

The main difference with Eq. (7) is that the normal vector $n_{j 0}^{S}$ is solidary with the movement of the mobile platform (see Fig. 2), so its orientation depends on the state of the mechanism, therefore $\delta$ is given by:

$\delta=\arccos \left(\frac{-\hat{L}_{i}(\varphi, \psi) \cdot\left[{ }^{0} \operatorname{Rot}_{p} \cdot n_{i 0}^{S}\right]}{\left\|-\hat{L}_{i}(\varphi, \psi)\right\| \cdot\left\|^{0} \operatorname{Rot}_{p} \cdot n_{i 0}^{S}\right\|}\right)$.

And should be verified that:

$|\delta| \leqslant \frac{\delta_{\max }}{2}$.

\subsubsection{Model for the collision between legs}

Any mechanism, and in particular those mechanisms that have closed loop kinematic structure suffer from possible autocollision among the links when the mechanism moves. Currently, there are several approaches that are used for the detection of collisions, some of them are simple and other are more complex and precise. A summary of them can be found in [18]. Usually these algorithms 


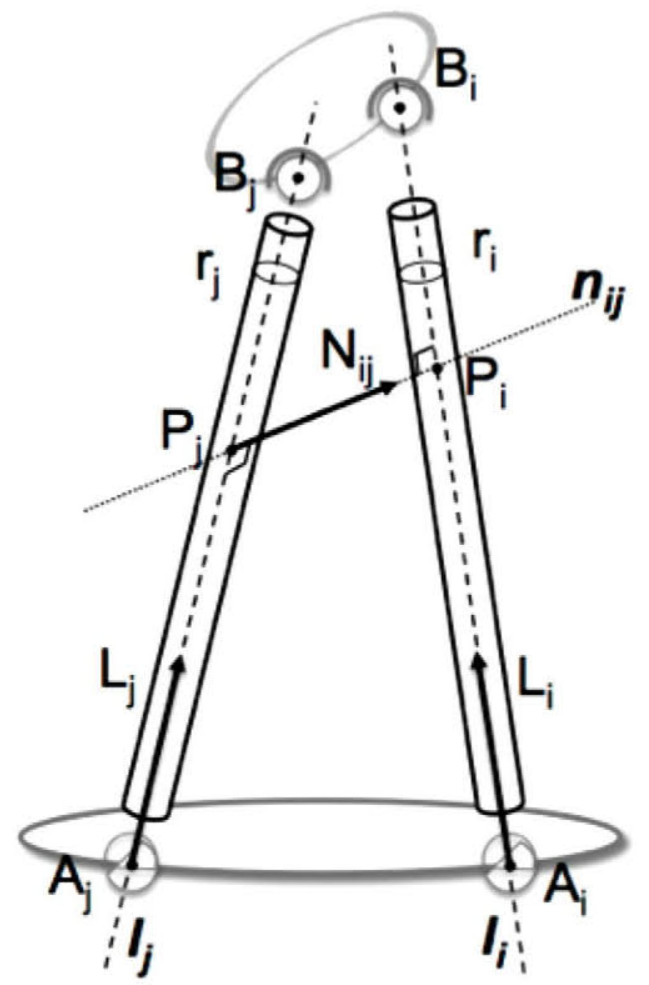

Fig. 3. Schematic diagram, used to detect collision between legs. $A_{i}, A_{j}, B_{i}, B_{j}$ are the points of insertion of the $i, j$ on the platforms lower and upper legs respectively $L_{j}$, $L_{i}$ are the vector addresses associated with the legs $i$ and $j . J_{j}$ is the vector direction of the normal line to the straight lines associated with the legs $i$ and j. $P_{i}, P_{j}$, are the points where it intersects the normal line with respective lines associated with the legs.

evaluate the minimum distance between the objects that belong to the mechanism analyzing them by pairs.

This work uses a simple algorithm that considers only the possible collisions between legs. Collisions between platforms and legs are not considered because they do not occur due to the constraint of the $U 1$ joint.

This algorithm considers each leg as a solid cylinder $\left(C_{i}\right)$ (Fig. 3) with radius $r_{i}$ and height $h_{i}$ given by (4). Each cylinder $\left(C_{i}\right)$ has a straight line $l_{i}$, whose direction vector is obtained from (3).

Whereas cylinders $\left(C_{i}\right)$ y $\left(C_{j}\right)$ for the legs $i$ and $j$ of the mechanism, said the cylinders collide if the minimum distance between the straight lines associated is greater than the sum of the radii of the cylinders. In other words,

$\Delta_{i j}>r_{i}+r_{j}$

where the distance $\Delta_{i j}$ represents the minimum distance between two straight lines, which is obtained from:

$\Delta_{i j}=\left(B_{j}-B_{i}\right) \cdot \hat{N}_{i j}$.

Being $N_{i j}$ the vector direction of the common straight line normal $\left(n_{i j}\right)$ to the straight lines $l_{i}$ and $l_{j}$ associated to the cylinders $C_{i}$ and $C_{j}$. The same is obtained from:

$\hat{N}_{i j}=\hat{L}_{i} \times \hat{L}_{j}$.

If Eq. (11) fails, this means that there is a possible collision between the legs. In this case points $P_{i}$ and $P_{j}$ corresponding to the intersection of the normal $n_{i j}$ line and the straight line $l_{i}$ and $l_{j}$ must be found. Then, we must verify that the points fall outside the segments defined by points $\overline{B_{i} A_{i}}$ and $\overline{B_{j} A_{j}}$, respectively. This is, considering the vector equations of the straight lines:

$$
\begin{aligned}
& l_{i}: P_{i}=A_{i}+\lambda_{i} \cdot \hat{L}_{i} \\
& l_{j}: P_{j}=A_{j}+\lambda_{j} \cdot \hat{L_{j}} \\
& n_{i j}: P_{i}=P_{j}+\lambda_{i j} \cdot \hat{N}_{i j}
\end{aligned}
$$

and obtaining a new expression according to the parameters of the three lines in:

$$
\left(A_{i}+\lambda_{i} \cdot \hat{L}_{i}\right)=\left(A_{j}+\lambda_{j} \cdot \hat{L}_{j}\right)+\lambda_{i j} \cdot \hat{N}_{i j} .
$$

Writing Eq. (15) in matrix form, you get the following system:

$$
\left[\begin{array}{lll}
\hat{L_{i}} & -\hat{L_{j}} & -\hat{N}_{i j}
\end{array}\right] \cdot\left[\begin{array}{lll}
\lambda_{i} & \lambda_{j} & \lambda_{i j}
\end{array}\right]^{T}=\left[\begin{array}{ll}
A_{j} & -A_{i}
\end{array}\right] .
$$

Solving Eq. (16), you can find the values of the parameters of the straight lines $\lambda_{i}, \lambda_{j}$ and $\lambda_{i j}$.

Therefore, $P_{i}$ are located outside of the segment defined by the points $\left(A_{i}, B_{i}\right)$, if

$0>\lambda_{i}$

$\lambda_{i}>\left\|L_{j}(\phi, \psi)\right\|$.

Similarly, $P_{j}$ are located outside of the segment defined by the points $\left(A_{j}, B_{j}\right)$, if

$0>\lambda_{j}$

$\lambda_{j}>\left\|L_{j}(\phi, \psi)\right\|$.

There will therefore be collision if at least one of expressions (17) or (18) is not satisfied. If $L_{i}$ and $L_{j}$ vectors are parallel, the distance between them shall be given by the distance between any two points belonging to a plane whose normal is parallel to both straight lines. In the particular case of this platform, the points $A_{i}$ and $A_{j}$ are considered, so:

$\left\|A_{j}-A_{i}\right\|<r_{i}+r_{j}$.

It is important to note that expressions (17)-(19), consider the distance between the lines associated with the legs of the mechanism, and not to the same volume of the legs. So, these expressions will be more accurate as the ratio of height/radius of the cylinder is greater. For this reason that it is necessary to include in the expressions a factor in security of agreement a $\lambda>k\left\|L_{i}\right\|$.

This work found experimentally that the factor of safety must be $\mathbf{k}=\mathbf{1 . 3}$, due to the geometric characteristics of the mechanism.

\subsubsection{Model of the open universal joint}

Universal joint used in this device is open type [19], in such a way that maximizes the angle allowed by the articulation.

Because the most influential factor in the workspace of the mechanism will be directly linked to the movement of open universal joint capabilities, the model should be as close to the real joint as we can.

CAD tools were used to reproduce the mechanism of open universal joint and rotations admissible on each of the axes were obtained with the same tools. These results have been plotting in Fig. 4. The figure shows curves that limit the range of movement of the joint. These curves are defined by polynomials. The coefficients of the polynomials defining curves are summarized in Table 1 (Fig. 4).

\subsection{Differential analysis}

Singularity analysis is carried out evaluating the Jacobian matrix of the mechanism. Jacobian matrix links the joint velocities with the Cartesian velocity of the end effector. In other words, if you define $\mathbf{q}$ as an state vector of the joint space (prismatic actuators), and $\mathbf{x}$ as a vector that describes the orientation of 


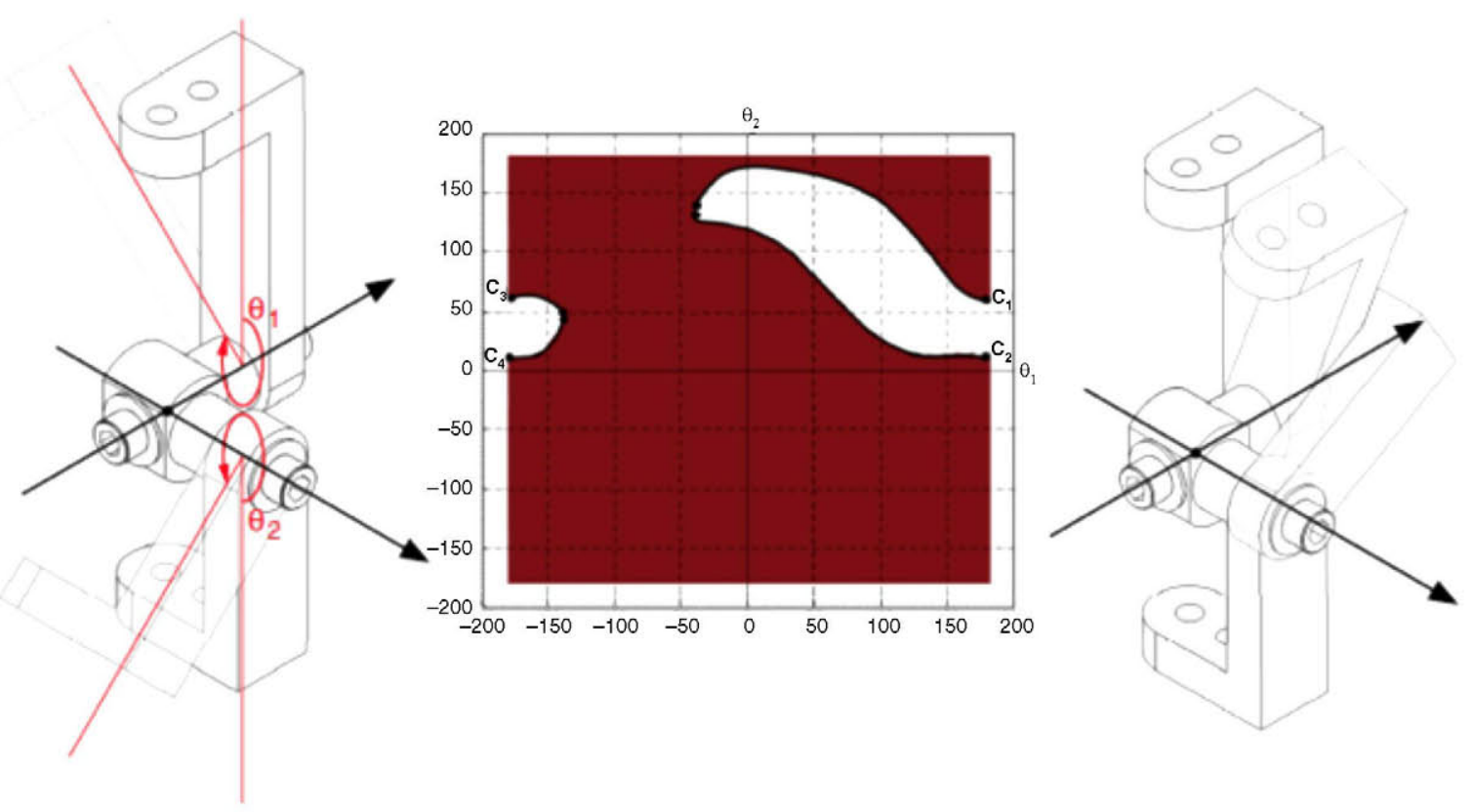

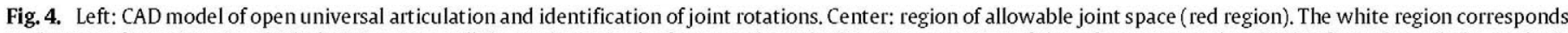

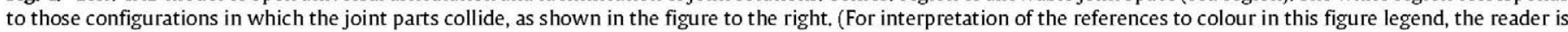
referred to the web version of this article.)

Table 1

Coefficients of the polynomials that limit the eligible region of work for the universal joint open. $F(x)=a_{5} * x^{5}+a_{4} * x^{4}+a_{3} * x^{3}+a_{2} * x^{2}+a_{1} * x+a_{0}$.

\begin{tabular}{|c|c|c|c|c|c|c|}
\hline Curve & $a 5$ & $a 4$ & $a 3$ & $a 2$ & $a 1$ & $a 0$ \\
\hline$c 1$ & $-4.145 e-09$ & $1.304 \mathrm{e}-06$ & $-6.062 e-05$ & -0.008557 & -0.3609 & 118.6 \\
\hline$c 2$ & $5.523 e-09$ & $-1.839 e-06$ & 0.0001822 & -0.00919 & 0.06183 & 171.2 \\
\hline$c 3$ & - & - & 0.0008329 & 0.4329 & 75.02 & 4346 \\
\hline$c 4$ & - & - & - & -0.01662 & -5.559 & -402.2 \\
\hline
\end{tabular}

the platform. The kinematic constraints imposed by the limbs are expressed as $\mathbf{f}(\mathbf{x}, \mathbf{q})=\mathbf{0}$. Differentiating with respect to time, a relation between the input joint rates and the end-effector output velocity is obtained:

$$
\mathbf{J}_{\mathbf{x}} \dot{\mathbf{x}}=\mathbf{J}_{\mathbf{q}} \dot{\mathbf{q}}
$$

where $\mathbf{J}_{\mathbf{x}}=\frac{\delta \mathbf{f}}{\delta \mathbf{x}}$ and $\mathbf{J}_{\mathbf{q}}=-\frac{\delta \mathbf{f}}{\delta \mathbf{q}}$.

Hence, the overall Jacobian matrix, J, can be written as:

$$
\dot{\mathbf{q}}=\mathbf{J} \dot{\mathbf{x}}
$$

where $\mathbf{J}=\mathbf{J}_{\mathbf{q}}^{-1} \mathbf{J}_{\mathbf{x}}$.

\subsubsection{Computational model of the screw-based Jacobian}

According to (21), the Jacobian matrix of the mechanism relates the velocity joints with the speed of the end effector. In a parallel mechanism, the speed of the end effector can be represented by a twist $\$_{p}$ resulting from:

$\$_{p}=\sum_{j=1}^{n} \dot{q}_{i, j} \cdot \hat{\$}_{i, j}=\left[\begin{array}{c}\omega_{n} \\ \nu_{0}\end{array}\right]$

where:

- $\hat{\$}_{i, j}: j$-th unit Twist linked to the $j$-th articulation of the $i$-th leg of the mechanism

- $\dot{q}_{i, j}$ : intensity of unitary Twist

- $\omega_{n}$ : angular velocity of the end effector

- $v_{0}$ : linear velocity of a point belonging to the end effector that is instantaneously coincident with the fixed reference system's origin.
The expression (23) includes all the Twist involved in the kinematics of the mechanism, actuated joints as well as passive ones. Applying the concept of orthogonal product in order to reduce the degree of complexity of the expression, twists associated with passive joints can be eliminated. Without going into details, the reciprocal screw $\$$ to a given Twist $\$$ is the one that satisfies the condition of reciprocity given by:

$\$$ $\$$ ○ $=0$.

In our case, we must look for the $\mathbf{g}$ reciprocal screws needed to eliminate the $\mathbf{k}$ passive joints. Then the orthogonal product is carried out on both sides of the expression (23) and the next expression is obtained for $i=1, \ldots, m$ leg:

$$
\left[\$_{r_{1, i}}^{T}, \$_{r_{2, i}}^{T}, \ldots, \$_{r_{g, i}}^{T}\right] \circ \$_{p}=\left[\$_{r_{1, i}}^{T}, \$_{r_{2, i}}^{T}, \ldots, \$_{r_{g, i}}^{T}\right] \circ \sum_{j=1}^{n} \dot{q}_{i, j} \dot{\hat{\$}}_{j, i}
$$

Noting the similarity with the expression (20) for each leg, next relation is obtained for $i=1, \ldots, m$ leg:

$\mathbf{J}_{\mathbf{x}, \mathbf{i}} \cdot \$_{\mathrm{p}}=\mathbf{J}_{\mathbf{q}, \mathbf{i}} \cdot \dot{\mathbf{q}}_{\mathbf{i}}$

Without losing generality, and considering that passive joints are from the $n-k+1$ of the kinematic chain joint, you can write:

$\mathbf{J}_{\mathbf{x}, \mathbf{i}}=\left[\begin{array}{c}\$_{r_{1, i}}^{T} \\ \$_{r_{2, i}}^{T} \\ \vdots \\ \$_{r_{g, i}}^{T}\end{array}\right]$ 


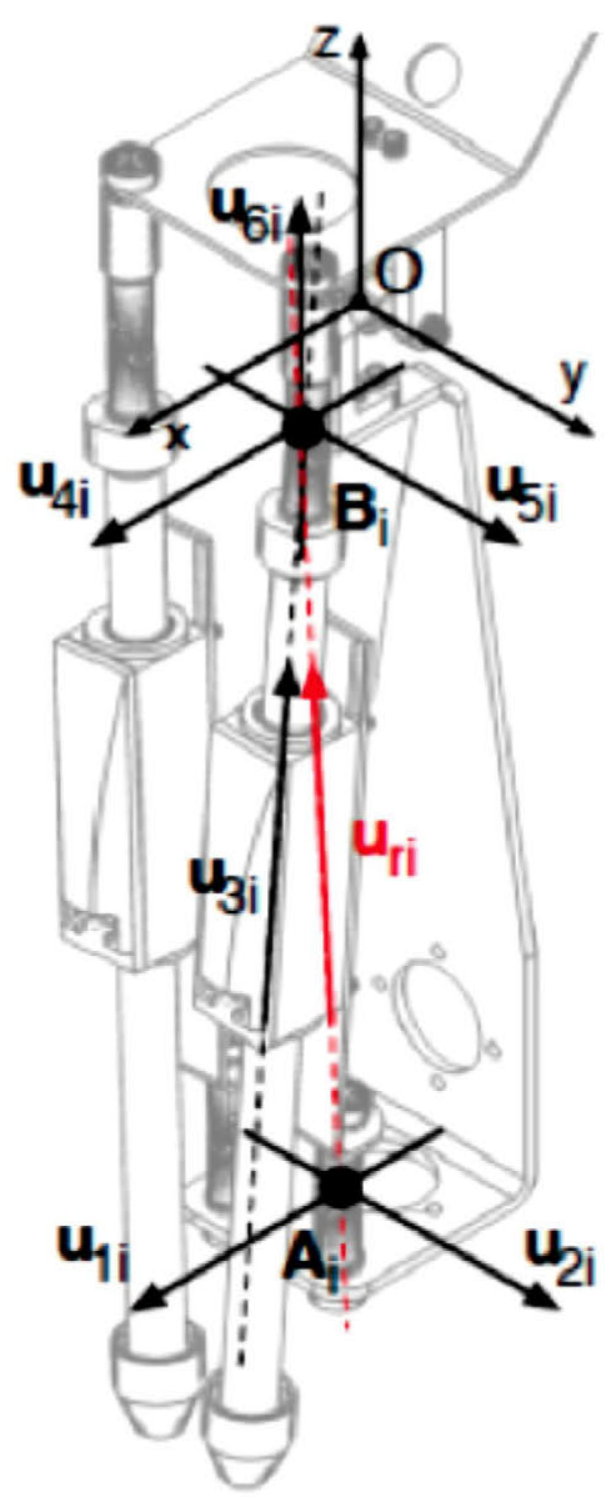

Fig. 5. Schematic drawing showing vector direction used for the screw-based Jacobian matrix calculation.

$\mathbf{J}_{\mathbf{q}, \mathbf{i}}=\left[\begin{array}{cccc}\$_{r_{1, i}}^{T} \circ \$_{1, i} & \$_{r_{1, i}}^{T} \circ \$_{2, i} & \cdots & \$_{r_{1, i}}^{T} \circ \$_{n-k, i} \\ \$_{r_{2, i}}^{T} \circ \$_{1, i} & \$_{r_{2, i}}^{T} \circ \$_{2, i} & \cdots & \$_{r_{2, i}}^{T} \circ \$_{n-k, i} \\ \vdots & \vdots & \ddots & \vdots \\ \$_{r_{g, i}}^{T} \circ \$_{1, i} & \$_{r_{g, i}}^{T} \circ \$_{2, i} & \cdots & \$_{r_{g, i}}^{T} \circ \$_{n-k, i}\end{array}\right]$

Therefore writing the expression (25) for each leg and expressing it on matrix notation, we get $J_{x} \cdot \$_{p}=J_{q} \cdot \dot{q}$, where:

$\mathbf{J}_{\mathbf{x}}=\left[\begin{array}{c}\mathbf{J}_{\mathbf{x}, 1} \\ \mathbf{J}_{\mathbf{x}, 2} \\ \vdots \\ \mathbf{J}_{\mathbf{x}, \mathbf{m}}\end{array}\right]$

$\mathbf{J}_{\mathbf{q}}=\left[\begin{array}{cccc}{\left[\mathbf{J}_{\mathbf{q}, 1}\right]} & 0 & \cdots & 0 \\ 0 & {\left[\mathbf{J}_{\mathbf{q}, 2}\right]} & \cdots & 0 \\ \vdots & \vdots & \ddots & \vdots \\ 0 & 0 & \cdots & {\left[\mathbf{J}_{\mathbf{q}, \mathbf{m}}\right]}\end{array}\right]$

In particular, for the mechanism presented in this paper, Fig. 5 shows that reciprocal screws associated with each joint are given by:

$$
\begin{aligned}
& \widehat{\$}_{1, i}=\left[\begin{array}{cc}
I & 0 \\
\tilde{a}_{i} & I
\end{array}\right]\left[\begin{array}{c}
u_{1, i} \\
0
\end{array}\right]=\left[\begin{array}{c}
u_{1, i} \\
\tilde{a}_{i} u_{1, i}
\end{array}\right] \\
& \widehat{\$}_{2, i}=\left[\begin{array}{cc}
I & 0 \\
\tilde{a}_{i} & I
\end{array}\right]\left[\begin{array}{c}
u_{2, i} \\
0
\end{array}\right]=\left[\begin{array}{c}
u_{2, i} \\
\tilde{a}_{i} u_{2, i}
\end{array}\right] \\
& \widehat{\$}_{3, i}=\left[\begin{array}{cc}
I & 0 \\
\tilde{a}_{i} & I
\end{array}\right]\left[\begin{array}{c}
0 \\
u_{3, i}
\end{array}\right]=\left[\begin{array}{c}
0 \\
u_{3, i}
\end{array}\right] \\
& \widehat{\$}_{4, i}=\left[\begin{array}{cc}
I & 0 \\
\tilde{b}_{i} & I
\end{array}\right]\left[\begin{array}{c}
u_{4, i} \\
0
\end{array}\right]=\left[\begin{array}{c}
u_{4, i} \\
\tilde{b}_{i} u_{4, i}
\end{array}\right] \\
& \widehat{\$}_{5, i}=\left[\begin{array}{cc}
I & 0 \\
\tilde{b}_{i} & I
\end{array}\right]\left[\begin{array}{c}
u_{5, i} \\
0
\end{array}\right]=\left[\begin{array}{c}
u_{5, i} \\
\tilde{b}_{i} u_{5, i}
\end{array}\right] \\
& \widehat{\$}_{6, i}=\left[\begin{array}{cc}
I & 0 \\
\tilde{b}_{i} & I
\end{array}\right]\left[\begin{array}{c}
u_{6, i} \\
0
\end{array}\right]=\left[\begin{array}{c}
u_{6, i} \\
\tilde{b}_{i} u_{6, i}
\end{array}\right]
\end{aligned}
$$

where $u_{k, i}$ represents a unit vector whose direction corresponds to the axis of the corresponding joint action, $a_{i}=\overline{A_{i} O}$ and $b_{i}=\overline{B_{i} O}$. Then the resulting end effector Twist becomes:

$$
\begin{aligned}
\$_{p}= & {\left[\begin{array}{c}
\omega_{e f} \\
0
\end{array}\right]=\omega_{1, i} \widehat{\$}_{1, i}+\omega_{2, i} \widehat{\$}_{2, i}+v_{3, i} \widehat{\$}_{3, i}+\omega_{4, i} \widehat{\$}_{4, i} } \\
& +\omega_{5, i} \widehat{\$}_{5, i}+\omega_{6, i} \widehat{\$}_{6, i}
\end{aligned}
$$

where $\omega_{j, i}$ and $v_{3, i}$ represent the twist intensities. Since the axis of all unactuated joints in each leg intersects the line passing through points $A_{i}$ and $B_{i}$, a unique screw that is reciprocal to all the unactuated joints is readily identified as:

$\widehat{\$}_{r, i}=\left[\begin{array}{c}u_{r, i} \\ \tilde{a}_{i} u_{r, i}\end{array}\right]$.

Therefore by applying orthogonal product on both sides of (36), you get:

$\widehat{\$}_{r, i}^{T} \circ \$_{p}=v_{3, i} \widehat{\$}_{r, i}^{T} \circ \$_{3, i}$

$$
\begin{aligned}
{\left[\begin{array}{c}
a_{i} \times u_{r, i} \\
u_{r, i}
\end{array}\right]^{T} \cdot\left[\begin{array}{c}
\omega_{e f} \\
0
\end{array}\right] } & =v_{3, i}\left[\begin{array}{c}
a_{i} \times u_{r, i} \\
u_{r, i}
\end{array}\right]^{T} \cdot\left[\begin{array}{c}
0 \\
u_{3, i}
\end{array}\right] \\
& =v_{3, i} u_{r, i}^{T} u_{3, i} .
\end{aligned}
$$

Writing 39 for the two legs containing actuated joints, you get:

$\left[\begin{array}{l}\left(a_{1} \times u_{r, 1}\right)^{T} \\ \left(a_{2} \times u_{r, 2}\right)^{T}\end{array}\right] \cdot\left[\omega_{e f}\right]=\left[\begin{array}{l}u_{r, 1}^{T} u_{3,1} \\ u_{r, 2}^{T} u_{3,2}\end{array}\right]\left[\begin{array}{l}v_{3,1} \\ v_{3,2}\end{array}\right]$.

Therefore:

$J_{x}=\left[\begin{array}{l}\left(a_{1} \times u_{r, 1}\right)^{T} \\ \left(a_{2} \times u_{r, 2}\right)^{T}\end{array}\right]$

$J_{q}=\left[\begin{array}{cc}u_{r, 1}^{T} u_{3,1} & 0 \\ 0 & u_{r, 2}^{T} u_{3,2}\end{array}\right]$.

\subsubsection{Singularity analysis}

Due to the existence of two Jacobian matrices $\left(\mathbf{J}_{\mathbf{x}}\right.$ and $\left.\mathbf{J}_{\mathbf{q}}\right)$, one can speak of a singularity of forward kinematics $\left(\operatorname{det}\left(\mathbf{J}_{\mathbf{x}}\right)=0\right)$ and singularities of inverse kinematics ( $\operatorname{det}\left(\mathbf{J}_{\mathbf{q}}\right)=0$ ). However, this work does not perform such differentiation and only considers that the mechanism is in a singular configuration when you check that $\operatorname{det}(\mathbf{J})=0$.

However, finding those configurations where it is verified that the determinant of the Jacobian matrix is zero requires a high computational cost. Therefore, we use an indirect method that consists on finding the value of the determinant of the Jacobian in all the configurations, then we note if this value is negative or positive. As it is known that the function of this 

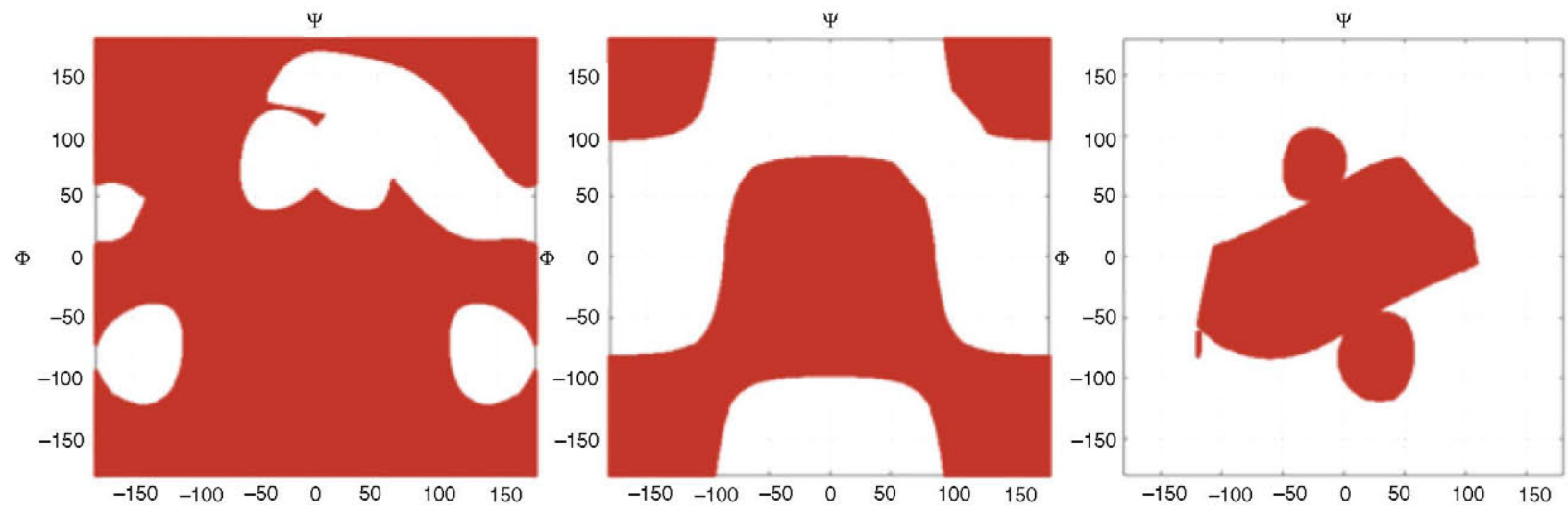

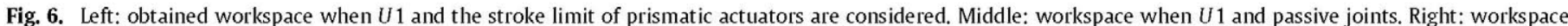
when $U 1$ constraint and autocollisions.

determinant is continue, therefore the border that separates the workspace in those configurations with positive determinant and negative determinant, corresponds to the configurations with zero determinant. This boundary defines a singular configuration.

\subsection{Dexterity analysis}

Another interesting information about the workspace is to evaluate the "quality" of the same. You can reach one point, so it belongs to your workspace, but you it is also important the dexterity or manipulability you have at this configuration. Salisbury and Angeles [20] defined the dexterity of a manipulator as the accuracy associated to the kinematic mechanism. They based its measure upon the condition number of the manipulator Jacobian matrix, where the Jacobian matrix maps the actuated joint velocities to the velocity of the moving platform in cartesian space. Mathematically, the quality of the performance of a robot with regard to the transmission of strength and speed can be obtained from the Jacobian matrix. It can be characterized by a measure called the condition number $C$, defined as:

$C(J)=\|J\| \cdot\left\|J^{-1}\right\|$

where

$\|J\|=\max _{x \neq 0} \frac{\|J \cdot x\|}{\|x\|}$

and $\|$ || is the Euclidean norm. However, as the number of condition can take values between $1 \leqslant C<\infty$, their inverse magnitude is normally used, the condition index $(\mathrm{CI})$ of the Jacobian matrix, whose value is bounded between $0<C I(J) \leqslant 1$. When the $C I$ is closer to 1 , it is said that the matrix $\mathbf{J}$ is well conditioned, i.e. located away from singularities. This index gives an idea of the local dexterity of the mechanism given a configuration. To get an idea of the global manipulation of the mechanism, the concept of the rate of Global condition (GCI) is used. This GCI is based upon the integration of the reciprocal of the condition number over the entire workspace. It is defined as:

$G C I=\frac{\int_{w} C I(\mathbf{J}) d w}{\int_{w} d w}$

where $w$ represents the set of points of the workspace.

\section{Results}

The results presented below were obtained by coding in Matlab ${ }^{(1)}$ the equations presented in Section 2. The geometrical parameters of the prototype are summarized in Table 2, and correspond to the geometric parameters of the first prototype build in the laboratories of the Universidad Politécnica de Madrid.
Table 2

Geometrical parameters of the 2UPS - $1 U$ platform.

\begin{tabular}{lll}
\hline \multirow{3}{*}{ Platform } & Mobile platform radius & $28.8 \mathrm{~mm}$ \\
& Fixed platform radius & $13.8 \mathrm{~mm}$ \\
& Distance between platforms & $210 \mathrm{~mm}$ \\
\hline Universal joint & Angle limit & $30^{\circ}$ \\
Spherical joint & Angle limit & $90^{\circ}$ \\
& $L_{\max }$ & $200 \mathrm{~mm}$ \\
Prismatic actuator & $L_{\min }$ & $0 \mathrm{~mm}$ \\
& Radius & $6 \mathrm{~mm}$ \\
\hline
\end{tabular}

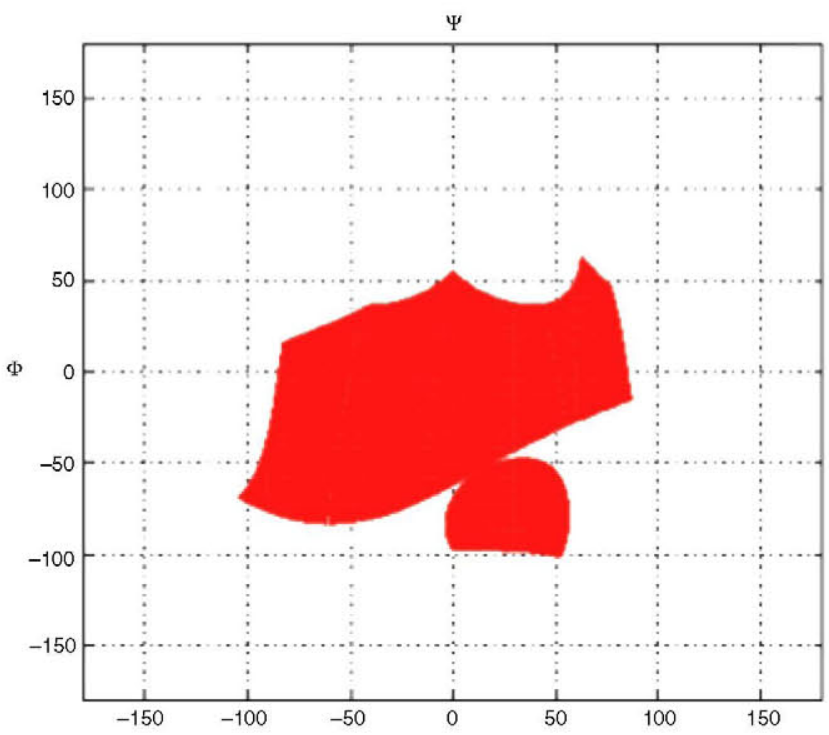

Fig. 7. Complete workspace of the $2 U P S-1 U$ mechanism.

\subsection{Results from the workspace analysis}

Fig. 6 shows the workspace of the mechanism considering separately the geometric restrictions modeled on Section 2. Left figure plots the workspace when only the $U 1$ constraint and the limit of the prismatic actuator are considered. Middle figure considers $U 1$ constraint and the passive joints, and right figure plots the results when consider $U 1$ constraint and the autocollision among legs.

To get the complete workspace, when all the constraints are taking account, we must intersect these three plots. This is done on Fig. 7. 


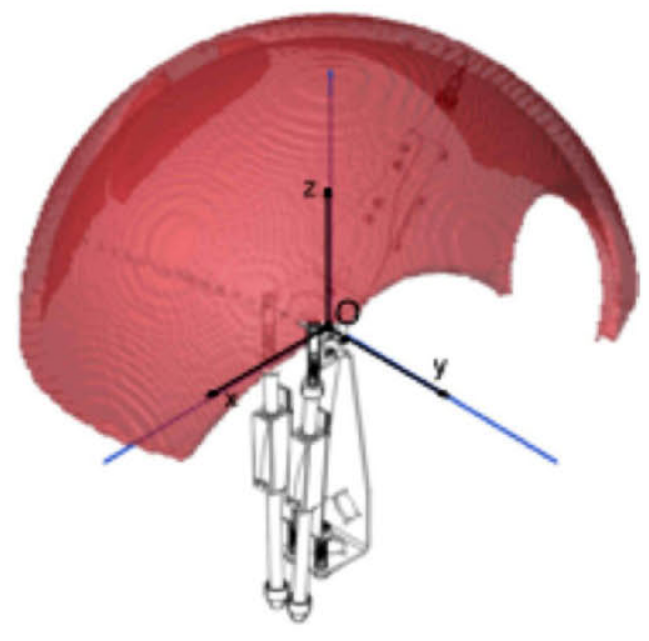

Fig. 8. Reachable workspace with a $15 \mathrm{~cm}$ length tool.

In Fig. 7, the workspace of the mechanism considering all geometric constraints. Results show that the maximum rotations of the mechanism are: $\psi_{\min }=-86^{\circ}, \psi_{\max }=86^{\circ}, \phi_{\min }=-60^{\circ}$, $\phi_{\max }=60^{\circ}$. This result is used to plan the movements of the robotic arm that supports this orientation device. To visually verify that this result allows the mechanism to be used on maxillofacial surgery, Fig. 8 presents possible positions that can be reached by $15 \mathrm{~cm}$ tool located in the center of rotation $U 1$. This Cartesian workspace represent the points that can be reached by this tool without moving the supporting robotic arm.

\subsection{Results from singularity analyses}

Fig. 9 plots the border where $\operatorname{det}(\mathbf{J})=0$. It can be observed that the singularity curve, defined by the border that divides the configurations whose determinant of the Jacobian matrix is positive or negative, falls out of the workspace presented on Fig. 7, so the workspace of the mechanism is free of singular configurations.

\subsection{Results from dexterity analyses}

A better understanding of the behavior of the device can be visualized on Fig. 10. This figure plots the $C I$. It can be observed that the mechanism is well conditioned within the workspace and

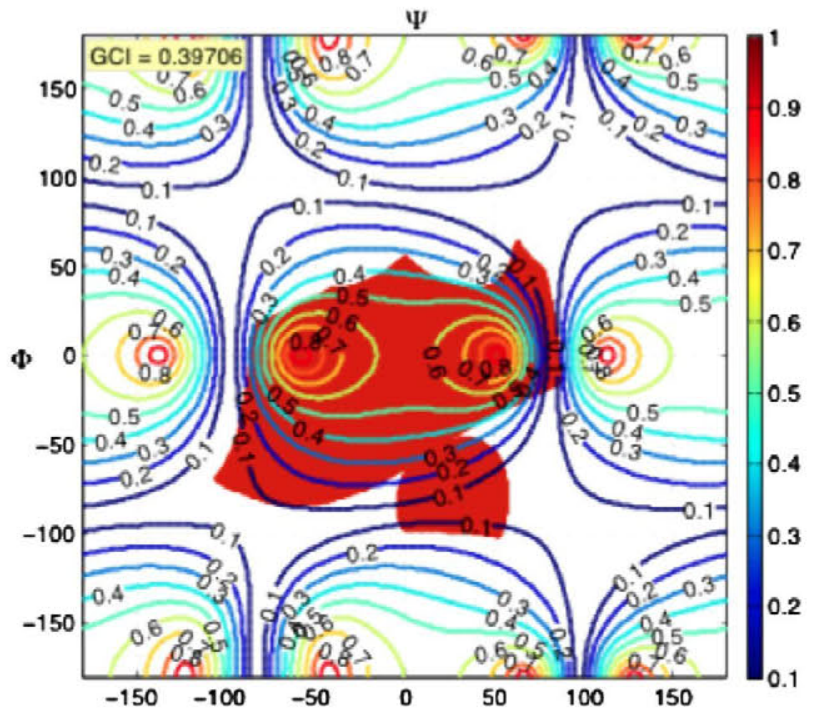

Fig. 10. Condition Index plot over workspace.

that the $\mathrm{CI}$ approaches to zero when going to the limits of the workspace. This conditioning of the Jacobian matrix, is getting worse as we move away from the center of the workspace.

\section{Conclusions}

The design steps and the methodology for the analysis of a new orientation device designed for maxillofacial surgery is presented. The workspace and the singularities inside have been deeply analyzed. The design gives a 2 dof spherical platform that has a workspace of $\Psi=[-100,80]$ and $\Phi=[-100,50]$, being $\Psi$ and $\Phi$ the rotation angles. Analyses have demonstrated that the wrist does not have singularities inside this workspace and that the dexterity of the mechanism is acceptable when evolves in this workspace. The results of these analyses had allowed to built a prototype of the wrist with the aim of using it as a wrist for bone drilling operations.

Currently, the first prototype is build, and it has been connected to a Phantom Omni haptic device to be controlled under a teleoperation scheme. A $15 \mathrm{~cm}$ length-milling tool has been located at the mobile platform, and the experiments of milling a bone and the experiment of following a trajectory on a virtual inferior jawbone has been carried out.
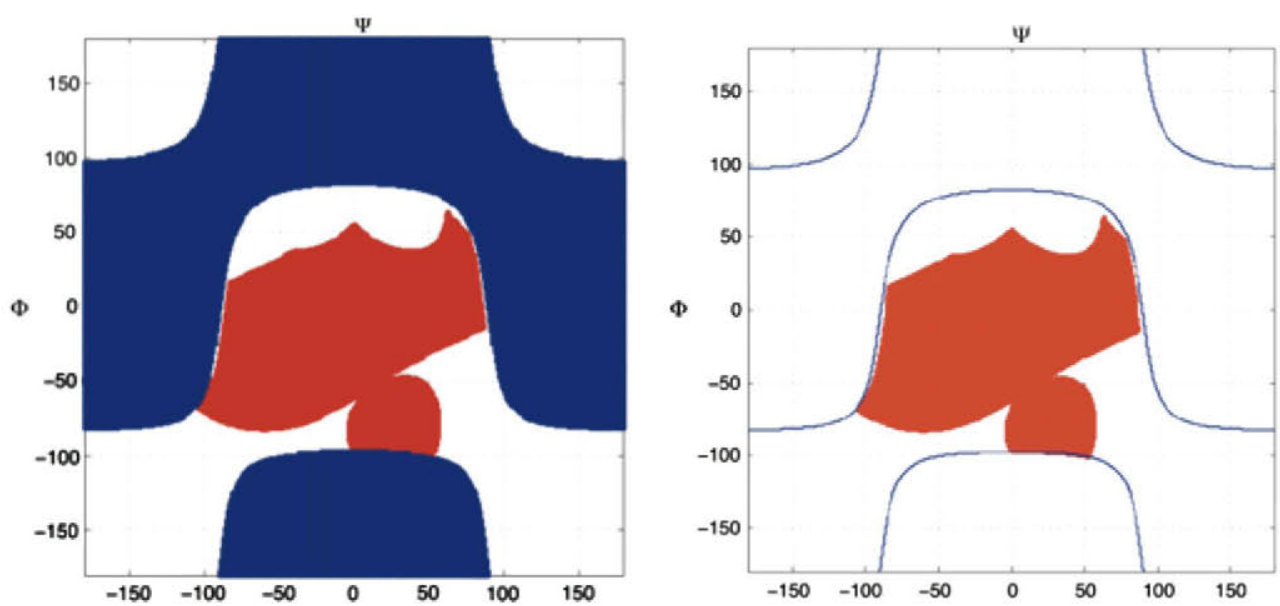

Fig. 9. Left: space of configurations where the determinant of the Jacobian matrix is negative. Right: singularity curves out of the workspace. 


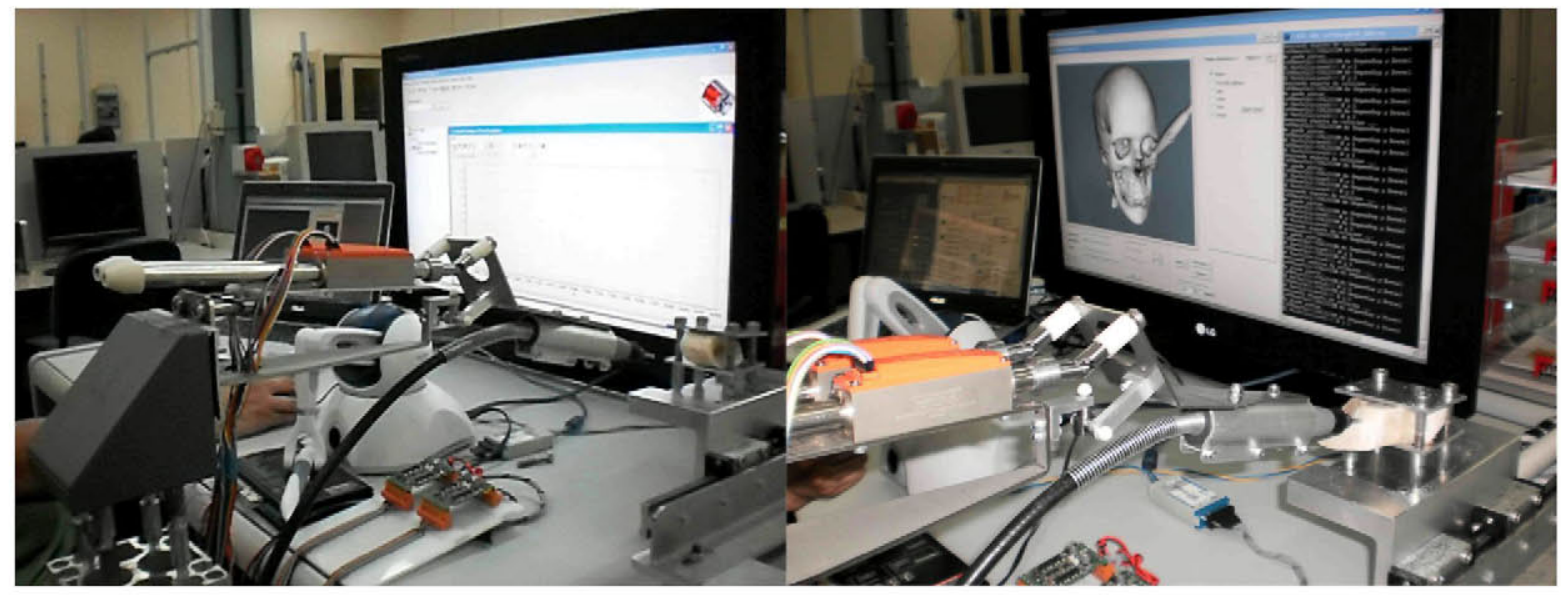

Fig. 11. Photographs of the device in the experiment of milling a bone.

The conclusions are that the developed design is able to cover the needed workspace for the jawbone surgery, and that the force requirements for doing a bone milling process are also fulfilled by the device. Fig. 11 shows photographs of the first prototype. The use of the open $U 1$ joint gives a wide orientation workspace.

Current prototype is hung up on a fixed platform. A video of the milling process of a bone is attached to this paper as multimedia additional material. Our next developments include integrating this orientation device on a positioning robotic arm, in order to simulate real procedures on maxillofacial surgery. To simulate these surgeries, current setup integrates a virtual environment able to build a 3D reconstruction of the patients' jawbone from the medical images, and to integrate the virtual representation of the milling tool located at the end of the orientation robot. This tool will allow us to virtually check the behavior of the robot in a wide series of surgeries.

\section{Acknowledgment}

Authors would like to thank the financial support of the Ministerio de Ciencia e Innovacion from Spain through the actions DPI2010-21126-C03-02, DPI2009-08778 and Robocity2030II-P2009/DPI1559.

\section{Appendix. Supplementary data}

Supplementary material related to this article can be found online at doi:10.1016/j.robot.2012.01.010.

\section{References}

[1] A. Lehman, K. Berg, J. Dumpert, N. Wood, A. Visty, M. Rentschler, S. Platt, S. Farritor, D. Oleynikov, Surgery with cooperative robots, Computer Aided Surgery 13 (2) (2008) 95-105.

[2] R. Galán, I. Moreno, R. Caballero, A. Jiménez, F. Matía, La Nariz electrónica: estado del arte, RIAII ISSN: 1697-7912, Vol. 6, No 3, Julio 2009, pp. 76-91.

[3] T. Fong, I. Nourbakhsh, K. Dautenhahn, A survey of socially interactive robots, Robotics and Autonomous Systems (ISSN: 0921-8890) 42 (3-4) (2003) 143-166. 10.1016/S0921-8890(02)00372-X.

[4] S.S Sastry, M Cohn, F Tendick, Milli-robotics for remote, minimally invasive surgery, Robotics and Autonomous Systems (ISSN: 0921-8890) 21 (3) (1997) 305-316. 10.1016/S0921-8890(96)00082-6.

[5] J.M. Sabater, C. Perez, N. Garcia, J.M. Azorin, M. Almonacid, Surgical engineering, Technology at surgery service Dyna, Ingeniería e industria Volumen: 85 no 3, pp: 225-236, abril 2010 ISSN: 0012-7361, Bilbao.

[6] E. Masatoshi, N. Seiji, Robotic surgery assisted by the ZEUS system, Endourooncology (2006) ISBN 978-4-431-21389-5.

[7] J. Moreno, C. Nuñez, M. Galante, S. Prieto, J. López, A. Silmi, Prostatectomica radical asistida por robot da Vinci: experiencia en el hospital clinico San Carlos, Laparoscopia y Robótica, Archivos españoles de urología (ISSN: 0004-0614) 61 (3) (2008) 385-396.

[8] R. Taylor, D. Stoianovici, Medical robotics in computer-integrated surgery, IEEE Transactions on Robotics and Automation 19 (5) (2003)
[9] Q. Hang, L. Zamorano, A. Pandya, R. Pérez, J. Gong, F. Díaz, The application accuracy of the neuromate robot-a quantitative comparison with frameless and frame-based surgical localization systems, Computer Aided Surgery 7 (2002) 90-98

[10] P. Kazanzides, G. Fichtinger, G. Hager, A.M. Okamura, L. Whitcomb, R.H. Taylor, Surgical and interventional robotics: part I, IEEE Robotics and Automation Magazine 15 (2) (2008) 122-130.

[11] Lung-Wen Tsai, Robot Analysis: The Mechanism of Serial and Paralle Manipulators, John Wiley \& Sons, 1999, pp. 20-21 (Chapter 1).

[12] J.M. Sabater, J.M. Azorin, R. Saltaren, R. Aracil, Magister-P; a 6-URS paralle haptic device with open control architecture, Robotica 23 (2) (2005) doi: $10.1017 /$ S0263574704000608.

[13] T. Nakano, N. Sugita, T. Ueta, Y. Tamaki, M. Mitsuishi, A parallel robot to assist vitreoretinal surgery, Springer Int J CARS (2009).

[14] F. Chan, I. Kassim, C. Lo, C. Long, D. Low, B. Ti, I. Ng, Image-guided robotic neurosurgery-an in vitro and in vivo point accuracy evaluation experimenta study, Surgical Neurology 71 (2009).

[15] L. Joskowicz, R. Shamir, M. Freiman, M. Shoham, E. Zehavi, F. Umansky, Y.Shoshan, Image-guided system with miniature robot for precise positioning and targeting in keyhole neurosurgery, Computer Aided Surgery (2006).

[16] Tzung-Cheng Tsai, Yeh-Liang Hsu, Development of a parallel surgical robot with automatic bone drilling carriage for stereotactic neurosurgery, IEEE SMC 2004, International Conference on Systems, Man and Cybernetics, October 2004, Hague, Netherlands.

[17] J.M. Sabater, N. Garcia, C. Perez, E. Fernandez, R. Saltarén, M. Almonacid, Kinematics Of A Robotic 3ups1s Spherical Wrist Designed For Laparoscopic Applications, in: International Journal of Medical Robotics and Computer Assisted Surgery, Vol. 6, John Wiley \& Sons, 2010, pp. 291-300.

[18] J.P. Merlet, D. Daney, Legs interference checking of parallel robots over a given workspace or trajectory, in: Proc. Of the 2006 IEEE Int. Conf. On Robotics and Automation, Orlando, Florida, May 2006.

[19] J.M. Sabater, R. Saltarén, R. Aracil, Robotics and Autonomous System in: Design, modelling and implementation Of A 6 URS parallel haptic device, vol. 47, Elsevier B.V, Netherlands, (ISSN: 0921-8890), 2004, pp. 1-10.

[20] J.K. Salisbury, J. Angeles, Articulated hands: force control and knematic issues, International Journal of Robotics Research 1 (1) (1982) 4-17.

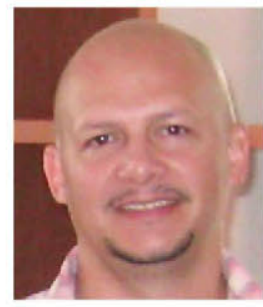

Jose Rolando Serracin Pitti received the B.S. degree in electronic engineering from the Universidad Tecnologica de Panama, Panama. He is currently working toward the Ph.D. degree at the Center for Automation and Robotics of the UPMCSIC, in Spain. His research interests include the kinematics, dynamics and control of robotic parallel mechanisms.

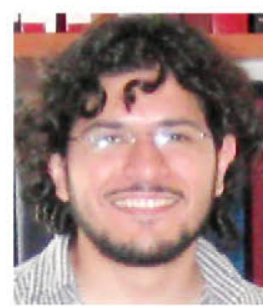

Lisandro J. Puglisi received the B.S. degree in electronic engineering in 2006 from the Universidad Nacional de Tucuman, Argentina, and the M.S. degree in Automation and Robotics at the Universidad Politecnica de Madrid, Spain, in 2010 . He is currently working toward the Ph.D. degree at the Center for Automation and Robotics of the UPMCSIC, in Spain. His research interests include the kinematics, dynamics and control of robotic paralle mechanisms. He has been an IEEE member since 2004. 


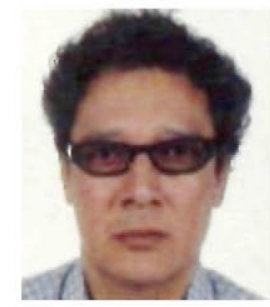

Roque Saltaren received the degree in mechanical engineering from the Universidad del Valle, Cali, Columbia in 1980, the M.Sc. degree in electrical engineering from the Universidad de los Andes, Bogota, Colombia in 1990, and the Ph.D. degree in industrial engineering from the Universidad Politecnica de Madrid, Madrid, Spain in 1996. Currently, he is an associate professor and a research scientist at the Group of Robots and Intelligent Machines, at Universidad Polite'cnica de Madrid, Escuela Tecnica Superior de Ingenieros Industriales, Madrid, Spain. He is a member of the IEEE.

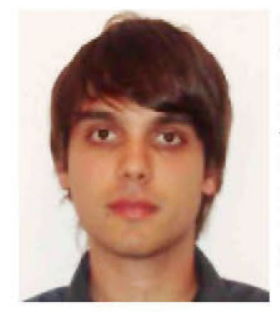

Gonzalo E. Ejarque received the B.S, degree in Mechatronics Engineering in 2010 from the École National d'Ingénieurs de Brest, France and the Universidad Nacional de Cuyo, Argentina. He received an M.S. degree in Automation and Robotics at the Universidad Politécnica de Madrid, Spain, in 2011. Currently, he is working toward the Ph.D. degree at the Center for Automation and Robotics of the UPM-CSIC, in Spain, His research interests include the design and development of robots and mechatronic systems. $\mathrm{He}$ is a member of the IEEE.

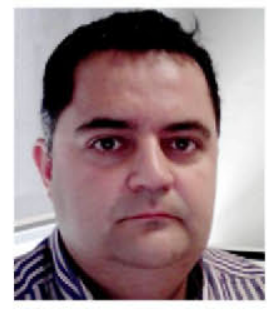

Jose M. Sabater-Navarro has a Ph.D. in engineering from the Universidad Miguel Hernandez (2003) and an M.Sc. in Industrial Engineering from the ETSII-Universitat Politecnica Valencia. Currently he is Sub-Director of the Politechnic School of Universidad Miguel Hernandez and Assistant Professor in robotics and computer vision at Miguel Hernandez University. He is a researcher at the Biomedical Neuro-engineering group. The main research areas include surgical robotics, computer assisted surgery, rehabilitation robots and robot design, and application of parallel kinematic structures to service robots.

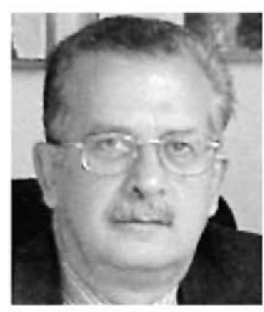

Rafael Aracil is Full Professor in the Departamento de $\mathrm{Au}$ tomatica, Ingenieria Electronica e Informatica Industrial of the Universidad Politecnica de Madrid. He received the Electrical Engineering degree in 1971 and the Doctor of Engineering degree in 1975 from this University. His main activities have been in the areas of Advanced Manufacturing, Robotics and Image Processing. Presently he is working on the development of robots for news applications and in intelligent teleoperation. In these areas he is making several realizations for different industrial enterprises. He is the author of several books, papers and communications in the cited topics. He is a member of several scientific and technical organizations besides being the leader of the IFAC Computer Vision Spanish Group and the Latin-American Robotics Network. He has worked in several EU funded projects (ESPRIT, BRITE and EUREKA). 